\title{
Automatic Image Matting of Synthetic Aperture Radar Target Chips
}

\author{
Benish AMIN ${ }^{1}$, M. Mohsin RIAZ2 ${ }^{2}$ Abdul GHAFOOR ${ }^{1}$ \\ ${ }^{1}$ National University of Science and Technology (NUST), Islamabad, Pakistan \\ ${ }^{2}$ COMSATS Institute of Information Technology, Islamabad, Pakistan \\ benishamin@students.mcs.edu.pk,mohsin.riaz@comsats.edu.pk, \\ abdulghafoor-mcs@nust.edu.pk
}

Submitted September 23, 2019 / Accepted December 27, 2019

\begin{abstract}
A matting technique to extract the targets from synthetic aperture radar (SAR) images is presented. Binary segmentation is performed initially for rough identification of target boundaries. Trimap is then estimated by combining the boundary structures of the input and segmented images using guided filter. In order to improve the accuracy of estimated trimap, super-pixels based segmentation is performed. A propagation based matting algorithm is then applied to separate the target from non-target region. Simulations conducted on different SAR images from MSTAR database show significance of proposed technique.
\end{abstract}

\section{Keywords}

Image matting, trimaps, SAR target detection, super-pixels

\section{Introduction}

Automatic extraction of targets from images (acquired from different sensors) play a key role in recognition and classification. Generally two types of data are used for that purpose i.e., optical and radar data. Figure 1 (a) shows the optical image, that comprises of visible wavebands and is similar to how human eye perceive the world. Whereas, the images acquired from synthetic aperture radar (SAR) sensors are of great importance due to their suitability in handling day-night operations and all-weather conditions. An example of X-band SAR image is shown in Fig. 1 (b). It can be observed that the speckle noise present in SAR image greatly affects the quality causing difficulty in interpretation.

Target extraction from SAR image requires 'target chip' that is obtained after detecting and discriminating the targets from SAR image. Target detection is performed on entire SAR image containing multiple targets and extracts the regions having potential target.

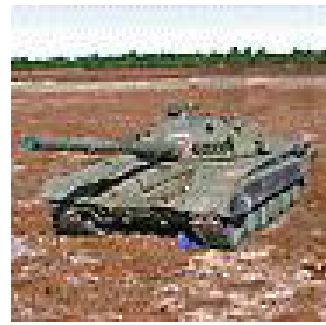

(a)

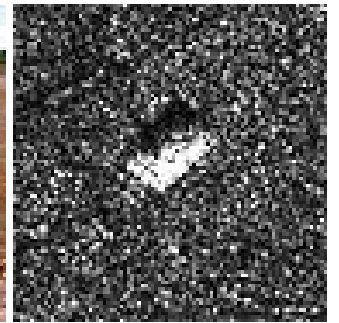

(b)
Fig. 1. Tank (T72), (a) Optical image, (b) X-band SAR image.

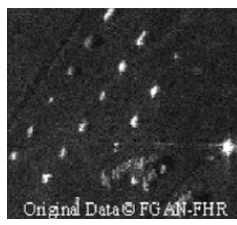

(a)

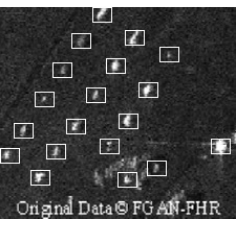

(b)

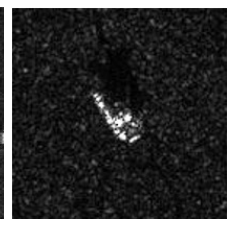

(c)
Fig. 2. (a) Original SAR scene, (b) Target detection (screening), (c) SAR target chip.

In case of stationary targets, screening techniques can be utilized to detect multiple targets from SAR scene whereas minimizing the false alarm [1]. After detection and discrimination of target regions, a target chip is acquired that contains background clutter and the actual target (along with its shadow) that lies at the centre of the chip [2]. This paper is intended to improve the accuracy of target extraction from its background clutter for effective target's recognition and classification. Figure 2 shows the screening process to identify potential targets and the target chip obtained by extracting region of interest.

In past decade, different methods have been developed for target extraction from SAR images. Samanta and Sanyal [3] proposed an adaptive threshold technique based on region merging to segment the SAR images. The technique produces satisfactory results in simple images but fails to perform well in complex background and noise conditions. Amoon and Rezai-Rad [4] and Tan, et al. [5] utilized mean filtering after histogram equalization to reduce the noise level. 


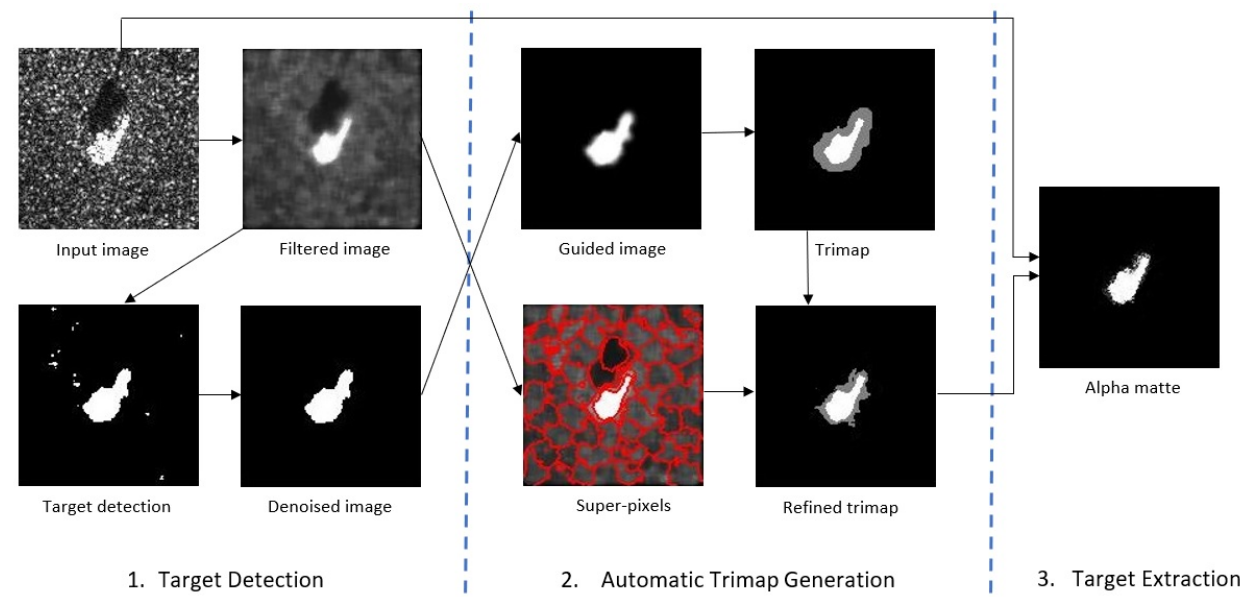

Fig. 3. Flowchart of proposed SAR target extraction technique.

Threshold value is estimated to detect binary target for efficient segmentation of SAR images. However the techniques fail to preserve useful spacial information and have limited performance for targets having several gray levels. Anagnostopulos [6] and Ding, et al. [7] applied basic thresholding and constant false alarm rate (CFAR) detection respectively, along with morphological operations for target region extraction, however the morphological operations fail to preserve target shape for complex targets.

Huang, et al. [8] proposed wavelet decomposition and CFAR for segmentation of the target and shadow regions from noisy SAR images. Han, et al. [9] proposed a level set approach where shape priors are used into active contours to extract the targets from SAR images, however the approach is inefficient since it requires user intervention to define the shape priors. R. Zhang and M. Zhang [10] utilized active contour without edges after histogram equalization to segment SAR targets, however the target boundaries are irregular for similar background color.

$\mathrm{Hu}$, et al. [11] adopted super-pixel segmentation technique based on statistical region merging [12] and nonsubsamples contourlet transform [13]. Fuzzy based clustering [14] is then used to estimate smooth target boundaries, however fails to completely mitigate the noise effects. Saliency based target extraction approaches [15], [16] utilize spectral residual and Bayesian-morphological operations, however the techniques provide limited performance for images containing noise. Ambrosanio, et al. [17] proposed Kolmogorov-Smirnov (KS) test based approach to detect the target, however it fails to discriminate the actual target and its shadow. Cho, et al. [2] proposed matting based approach to extract the target while preserving the intensity information. The approach provides robustness, however has limited performance for noisy images.

Concisely, existing techniques failed to accurately extract target from SAR target chip due to presence of noise, which made the interpretation and extraction of targets a challenging task. In this work, a matting technique for extraction of target from SAR target chips is proposed. Since the aim of this work is to separate the target region from non-target region, it can be assumed that the target chip is a combination of two layers i.e., target and non-target layer. However, in real-time, the intensity of SAR images varies due to intensity variation of the sensing environment caused by either target reflectance, scattering characteristics or coherent processing of SAR images. The varying intensity of the target area defines the opacity of the target, that can be estimated by image matting. (Matting aims to extract foreground regions from image by estimating the opacity value of pixels near boundary areas, that are mixed between foreground and background). Thus, image matting extracts the target area by assigning target as foreground, non-target as background and opacity of the target as alpha matte. The main contributions of proposed technique are described below,

1. The impact of noise is reduced using median filtering for correct identification of target region.

2. Trimaps are estimated automatically, and their accuracy is improved via super-pixels based segmentation approach.

3. Accurate target is extracted from target chip using propagation based matting approach.

\section{Proposed Methodology}

Image matting deals with the problem to separating foreground objects from background by estimating the opacity values of the foreground objects. Since it is an illposed problem, thus user intervention is required to generate a trimap (to divide the image into foreground, background and unknown regions). However, user interaction is difficult and time-consuming while processing SAR target chips. The paper proposed an automatic trimap generation technique to solve this problem (as represented in the flowchart shown in Fig. 3). For that purpose, correct identification of target region is required. Let $G$ be the input SAR image chip with 


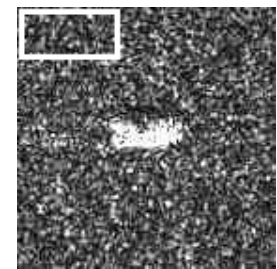

(a)

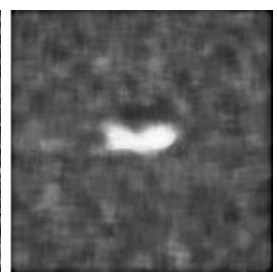

(b)

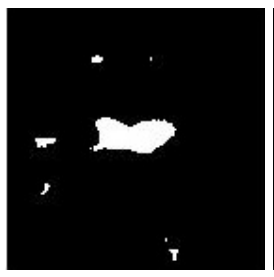

(c)

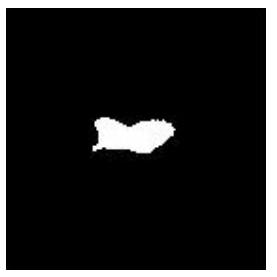

(d)

Fig. 4. Binary segmentation for initial target detection, (a) selected patch to calculate mean and standard deviation, (b) thresholding results, (c) target area detection, (d) target area noise removal.

dimensions $m \times n$. The first step is to apply median filter to mitigate the effect of speckle noise, that limits the ability to detect ground targets,

$$
M=\xi(G, \varphi)
$$

where $\xi$ indicates median filtering and $\varphi$ represents filter size $(8 \times 8)$. Decreasing the filter size reduces some amount of noise but unable to completely removed it, while increasing the filter size over-smooths the image.

In SAR images, the target region has high mean intensity value as well as high standard deviation (due to increase in variability of pixel intensities caused by edges and scattering centres). Based on these characteristics, a patch from the corner of $G$ representing the background area, is selected (since the target is roughly present at image centre) to identify the actual target area. The threshold value is computed as,

$$
t=\mu+k \delta
$$

where $\mu$ and $\delta$ represents the mean and standard deviation of patch, respectively, and $k=0.9$ is the bias.

The threshold value is applied on each pixel of image $M$ to estimate binary target image $B$ i.e.,

$$
B(m, n)=\left\{\begin{array}{lll}
1 & \text { if } & M(m, n)>t \\
0 & \text { if } & M(m, n) \leq t
\end{array}\right.
$$

The threshold correctly identifies the foreground target but also produces some amount of noise in non-target region. Thus, morphological opening operation is used to remove unwanted artifacts and noise;

$$
H=\left(B \ominus d_{1}\right) \oplus d_{2}
$$

where $\ominus$ and $\oplus$ represents erosion and dilation operators respectively with disk structure elements $d_{1}$ and $d_{2}$. Figure 4 shows the result of binary segmentation for initial target detection.

After the identification of target and non-target areas, a trimap is generated by passing the binary mask $H$ and guidance image $M$ through guided filter i.e.,

$$
F \longleftarrow \text { Guided filter }(H, M, \omega)
$$

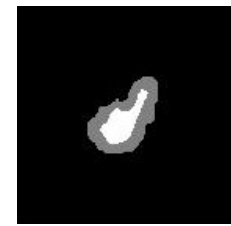

(a)

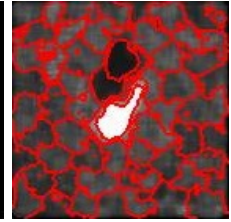

(b)

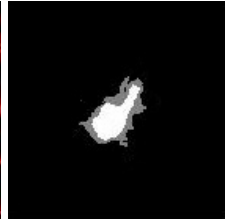

(c)
Fig. 5. Automatic trimap generation (a) estimated trimap using guided filtering (b) super-pixels based segmentation of denoised image (c) refined trimap by mapping superpixels based segmentation.

where $\omega$ represents local window size of the guided filter. The initial trimap is defined as,

$$
T_{\mathrm{o}}(m, n)=\left\{\begin{array}{ccr}
1 & \text { if } & F(m, n)=1 \\
0 & \text { if } & F(m, n)=0 \\
0.5 & & \text { otherwise }
\end{array}\right.
$$

where $T_{\mathrm{o}}(m, n)=1$ represents the foreground region, $T_{\mathrm{o}}(m, n)=0$ represents the background region and $T_{\mathrm{o}}(m, n)=0.5$ defines the unknown region.

To further refine the trimap by reducing the unknown region, the image $M$ is passed through simple linear iterative clustering algorithm (SLIC) [18], that groups the image pixels on the basis of color and texture similarities and represent them as super-pixels. i.e., (Fig. 5(b)).

$$
\chi \longleftarrow \text { SLIC }(M(m, n), \vartheta)
$$

where $\vartheta$ denotes the total number of super pixels. Let $\chi^{(i)}$ be the $i^{\text {th }}$ super pixel (where $i=1,2,3, \ldots, \vartheta$ ) and $T_{\mathrm{o}}^{(i)}(m, n)$ represents the pixels of trimap in $i^{\text {th }}$ super pixel, the improved trimap $T^{(i)}(m, n)$ is defined as,

$$
T^{(i)}(m, n)=\left\{\begin{array}{ccc}
1 & \text { if } & \bar{T}_{\mathrm{o}}^{(i)}(m, n)>\gamma_{1} \\
0 & \text { if } & \bar{T}_{\mathrm{o}}^{(i)}(m, n)<\gamma_{2} \\
0.5 & \text { otherwise } &
\end{array}\right.
$$

where $\bar{T}_{\mathrm{o}}^{(i)}(m, n)$ represents the mean value of pixels belong to $i^{\text {th }}$ super-pixel of initial trimap $T_{\mathrm{o}} \cdot \gamma_{1}=0.8$ and $\gamma_{2}=0.2$ are empirically selected constants with $\gamma_{1}>\gamma 2$. Increasing the threshold $\gamma_{1}$ causes many definite foreground pixels to be marked as unknown, whereas lowering the threshold $\gamma_{1}$ may cause unknown pixels to be part of definite foreground. 


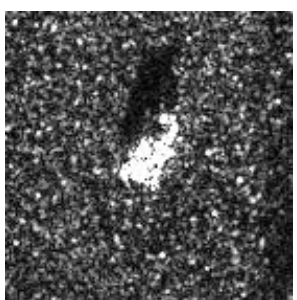

(a)

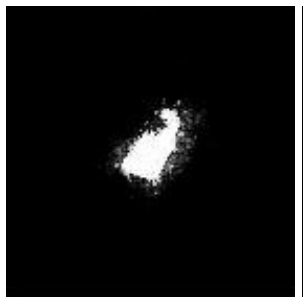

(f)

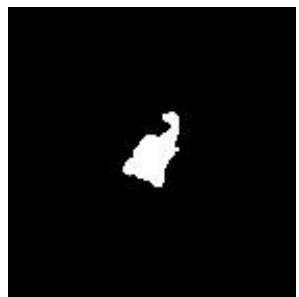

(b)

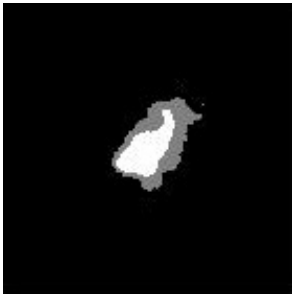

(g)

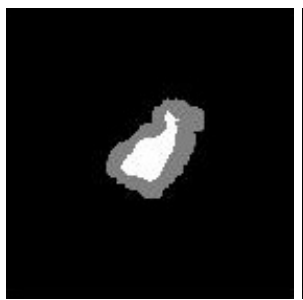

(c)

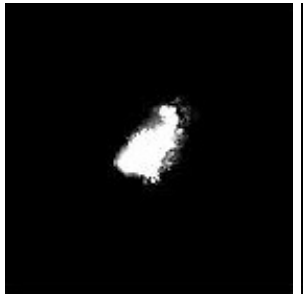

(h)

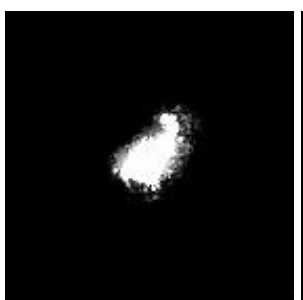

(d)

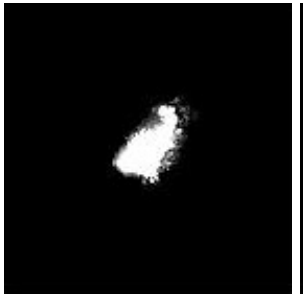

(i)

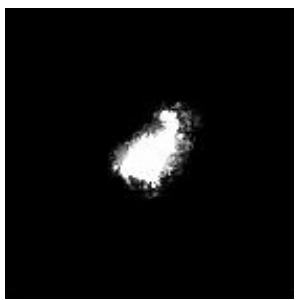

(e)

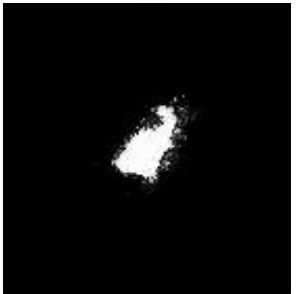

(j)

Fig. 6. Matting results, (a) input SAR image, (b) ground truth, (c) trimap without super-pixels based refinement, (d) CFM [19], (e) KL-Matting [20], (f) KNN-Matting [21], (g) proposed trimap, (h) CFM [19] with proposed refined trimap, (i) KL-Matting [20] with proposed refined trimap, (j) KNN-Matting [21] with proposed refined trimap.

Similarly, Increasing the threshold $\gamma_{2}$ causes many unknown pixels to be part of background region, while lowering the threshold $\gamma_{2}$ leaves many definite background pixels as unknown.

The final trimap $T(m, n)$ is obtained by assigning the value 1 to the pixels of trimap that belong to super-pixel $i$, if their mean value is greater than defined threshold $\gamma_{1}$. Similarly, 0 value is assigned to the pixels of trimap that are part of super-pixel $i$, if there mean value is less than defined threshold $\gamma_{2}$ and 0.5 is assigned to all the remaining pixels in the super-pixel region.

The target chip consists of a target region (that is a connected region with no holes) and the background clutter. Based on these characteristics, propagation based matting is applied to define affinities between neighbouring pixels based on intensity similarity instead of sampling based matting, that is useful for non-distributed color distribution. Knearest neighbours (KNN) matting algorithm [21] is applied on source image $G$ with refined trimap $T$ to generate alpha matte (which separates the target and non-target regions) i.e.,

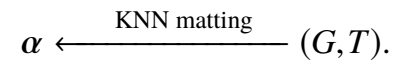

\section{Results and Discussion}

The proposed technique is simulated using publicly available MSTAR database [22] containing X-band SAR images. The images are captured with different dispersion angles and orientation, with target that lies at the centre of the image. The targets don't have clear edges, and the presence of speckle noise in SAR images make the target detection and extraction a more challenging task.
The simulations are conducted on an Intel-Core i7-3520M CPU (2.90GHz) with 8-GB RAM and MATLAB R-2014a. The proposed technique is analyzed visually and quantitatively, by applying different matting techniques including closed form matting (CFM) [19], Kullback-Leibler divergence based sparse matting (KL sparse matting) [20] and KNN matting [21]. Figure 6 shows the benefit of superpixels based trimap refinement step. Figure 6 (c) shows the estimated trimap using guided filtering, whereas, the matting by applying CFM [19], KL sparse matting [20] and KNN matting [21] are shown in Fig. 6 (d),(e),(f) respectively. The results clearly show the amount of error around the boundaries of the target. However, matting results (shown in Fig. 6 (h),(i),(j)) produced after refining the trimap using super-pixels (Fig. $6(\mathrm{~g})$ ) are much improved, consequently enhancing the target extraction accuracy.

The comparison of different matting techniques with proposed trimap is shown in Fig. 7. In CFM [19], $\alpha$ is estimated by pixels affinities based on color line model. KL sparse matting [20] collects sparse set of definite foreground/background samples to compute the value of unknown pixel. KNN matting [21] is based on non-local principle which utilizes closed-form solution to find pixel affinities in k-nearest neighbours. Figure 7 (a) shows the input target chips containing the targets that lie at the center of the chip along with the background clutter. The ground truths indicating the actual target with removed background are shown in Fig. 7 (b). The performance of different matting techniques is analyzed to extract targets from SAR images. It can be observed that all the matting techniques successfully extract the target without losing any information due to accurate trimap estimation. However, CFM [19] produces over-smooth results along the boundaries of the extracted target. Moreover, KL sparse matting [20] fails to completely overcome noise artifacts from background, thus produces inaccurate results. 

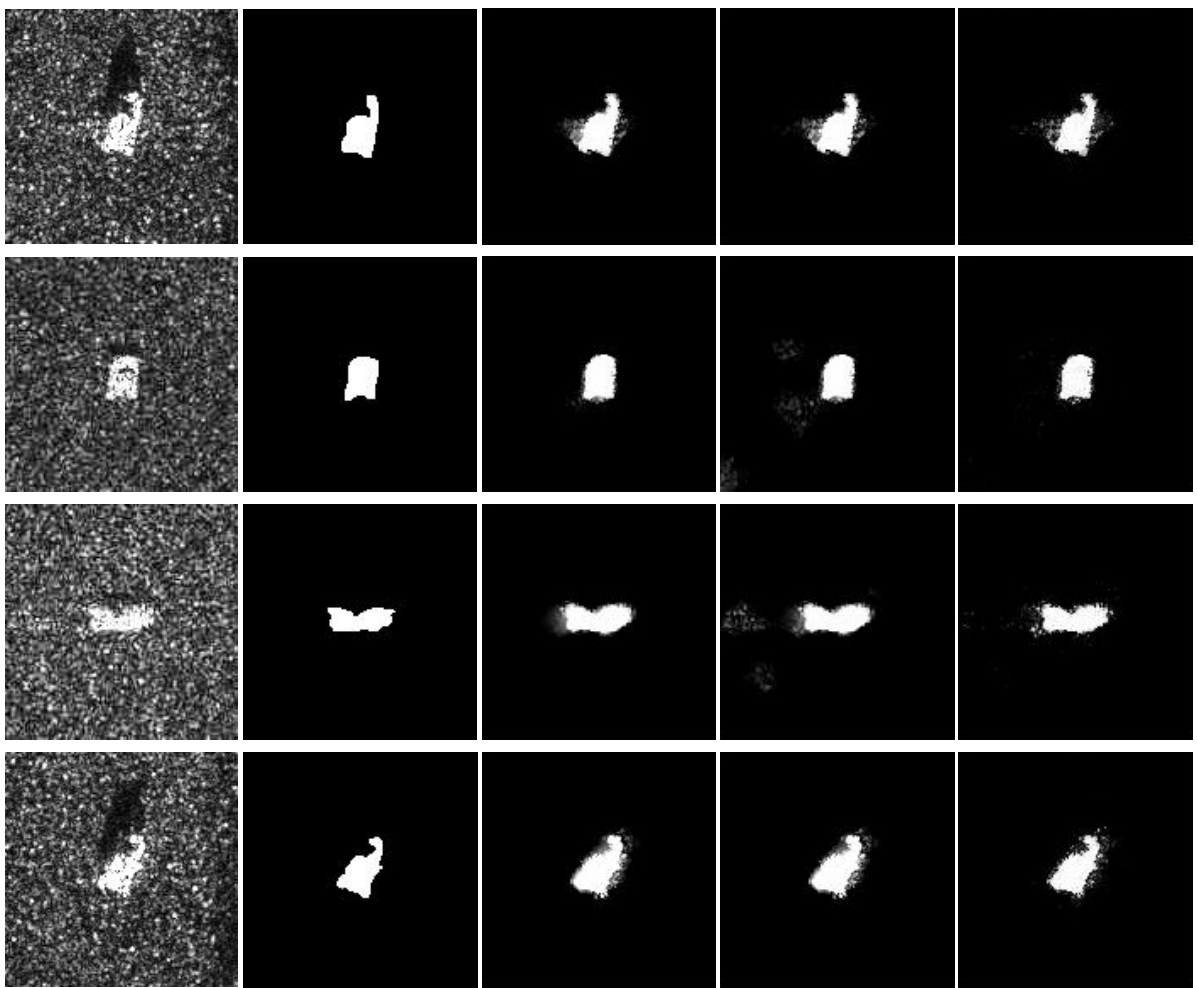

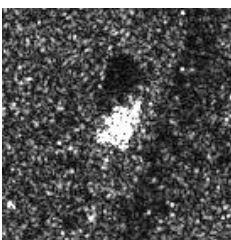

(a)

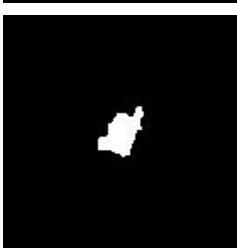

(b)

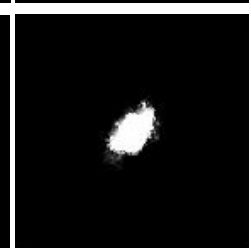

(c)

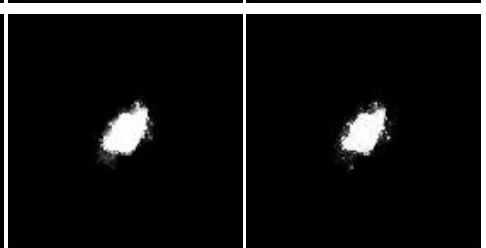

(d) (e)

Fig. 7. Comparison of matting techniques (a) input SAR image (b) ground truth (c) CFM [19] with proposed refined trimap (d) KL-Matting [20] with proposed refined trimap (e) KNN-Matting [21] with proposed refined trimap.

In comparison, KNN matting [21] better extracts the target with refined trimap without losing any target information and generating less noise along target boundaries.

Table 1 shows the confusion matrix of proposed technique against ground truth. Total 1000 images, that contain target along with its shadow and background clutter, are selected from MSTAR database. The proposed technique correctly extracted the target and non-target regions for 973 images. There were 20 cases where targets were incorrectly identified as non-targets, and for 7 cases some non-target region was incorrectly identified as target region. Therefore the recall score (ratio of actual targets that are correctly extracted) of proposed technique is $97.9 \%$.

The specificity (ratio of non-target regions that are correctly identified as non-target) is equal to $99.2 \%$. The precision (positive predictive value) of the proposed technique is $99.2 \%$ and the negative predictive value is equal to $97.9 \%$. The overall accuracy of proposed technique is $98.6 \%$.

To verify the accuracy of extracted target maps, mean square error (MSE) and peak signal to noise ratio (PSNR) are computed by comparing matting results with ground truths.
The low values of MSE and high values of PSNR indicate good results. The average numerical results evaluated on MSTAR database shown in Tab. 2 as well as the individual results in Tab. 3 clearly show that extracted target boundaries are more accurate with proposed refined trimaps as compared to other techniques.

\begin{tabular}{|l|l|l|l|l|}
\hline \multirow{2}{*}{ Total images: 1000} & \multicolumn{2}{|c|}{ Ground truth } & \multirow{2}{*}{} \\
\cline { 3 - 4 } \multicolumn{2}{|c|}{ Output } & Target & Non-target & \\
\cline { 2 - 4 } & Non-target & 973 & 7 & $99.2 \%$ \\
\hline \multicolumn{2}{|c|}{} & $97.9 \%$ & 973 & $97.9 \%$ \\
\hline
\end{tabular}

Tab. 1. Confusion matrix of proposed technique.

\begin{tabular}{|l|l|c|l|}
\hline \multicolumn{2}{|c|}{ Proposed matting results } & MSE & \multicolumn{1}{c|}{ PSNR } \\
\hline \multirow{3}{*}{ without super-pixels } & CFM [19] & 0.0092 & 20.3621 \\
\cline { 2 - 4 } & KL Matting [20] & 0.0096 & 20.1772 \\
\cline { 2 - 4 } & KNN Matting [21] & 0.0068 & 21.6749 \\
\hline \multirow{3}{*}{ with super-pixels } & CFM [19] & 0.0065 & 21.8708 \\
\cline { 2 - 4 } & KL Matting [20] & 0.0067 & 21.73925 \\
\cline { 2 - 4 } & KNN Matting [21] & 0.0056 & 22.5182 \\
\hline
\end{tabular}

Tab. 2. Average quantitative analysis of matting techniques. 


\begin{tabular}{|c|l|c|c|c|c|c|c|}
\hline $\begin{array}{c}\text { Quantitative } \\
\text { metrics }\end{array}$ & \multirow{2}{*}{ Examples } & \multicolumn{2}{|c|}{ Proposed matting without super-pixels } & \multicolumn{2}{c|}{ Proposed matting with super-pixels } \\
\cline { 3 - 8 } & & $\begin{array}{c}\text { CFM } \\
{[19]}\end{array}$ & $\begin{array}{c}\text { KL-Matting } \\
{[20]}\end{array}$ & $\begin{array}{c}\text { KNN-Matting } \\
{[21]}\end{array}$ & $\begin{array}{c}\text { CFM } \\
{[19]}\end{array}$ & $\begin{array}{c}\text { KL-Matting } \\
{[20]}\end{array}$ & $\begin{array}{c}\text { KNN-Matting } \\
{[21]}\end{array}$ \\
\hline \multirow{4}{*}{ MSE } & Example 1 & 0.0094 & 0.0096 & 0.0072 & 0.0054 & 0.0057 & 0.0049 \\
\cline { 2 - 8 } & Example 2 & 0.0055 & 0.0068 & 0.0032 & 0.0025 & 0.0029 & 0.0022 \\
\cline { 2 - 8 } & Example 3 & 0.0072 & 0.0085 & 0.0048 & 0.0054 & 0.0060 & 0.0047 \\
\cline { 2 - 8 } & Example 4 & 0.0082 & 0.0080 & 0.0050 & 0.0065 & 0.0065 & 0.0049 \\
\cline { 2 - 8 } & Example 5 & 0.0061 & 0.0064 & 0.0044 & 0.0045 & 0.0045 & 0.0033 \\
\hline \multirow{4}{*}{ PSNR } & Example 1 & 20.2672 & 20.1923 & 21.4620 & 22.6760 & 22.4237 & 23.0957 \\
\cline { 2 - 8 } & Example 2 & 22.6139 & 21.8073 & 24.9657 & 26.0794 & 25.4954 & 26.7761 \\
\cline { 2 - 8 } & Example 3 & 21.4353 & 20.6893 & 23.2016 & 22.6679 & 22.1957 & 23.2157 \\
\cline { 2 - 8 } & Example 4 & 20.8687 & 20.9945 & 23.0323 & 21.8581 & 21.8629 & 22.9745 \\
\cline { 2 - 7 } & Example 5 & 22.1428 & 21.9638 & 23.6310 & 23.4587 & 23.5039 & 24.7632 \\
\hline
\end{tabular}

Tab. 3. Quantitative analysis of matting techniques.

\section{Conclusion}

A matting technique to extract the target from SAR target chips is proposed. Initially, the technique roughly identifies the target through binary segmentation. A trimap is then estimated by applying guided filter, which is further refined by performing super-pixels based segmentation. Finally, propagation based matting algorithm is applied to separate the target from non-target region. Visual and quantitative analysis is performed on different SAR images from MSTAR database to show the significance of proposed technique.

\section{References}

[1] MiddelmanN, W., EBerT, A., THOENNESSEN. A. Automatic target recognition in SAR images based on a SVM classification scheme. In International Conference on Adaptive and Natural Computing Algorithms. Berlin (Germany), 2007, p. 492-499. DOI: $10.1007 / 978-3-540-71629-7-55$

[2] CHO, H.-W., CHO, Y.-R., KIM, B.-K., et al. Image matting for automatic target recognition. IEEE Transactions on Aerospace and Electronic Systems, 2017, vol. 53, no. 5, p. 2233-2250. DOI: 10.1109/TAES.2017.2690529

[3] SAMANTA, D., SANYAL, G. An approach of segmentation technique of SAR images using adaptive thresholding technique. International Journal of Engineering Research and Technology, 2012, vol. 1, no. 7 , p. $1-4$

[4] AMOON, M., REZAI-RAD, G.-A. Automatic target recognition of synthetic aperture radar (SAR) images based on optimal selection of Zernike moments features. IET Computer Vision, 2014, vol. 8, no. 2, p. 77-85. DOI: 0.1049/iet-cvi.2013.0027

[5] TAN, J., FAN, X., WANG, S., et al. Target recognition of SAR images via matching attributed scattering centers with binary target region. Sensors, 2018, vol. 18, no. 9, p. 3019. DOI: 10.3390/s18093019

[6] ANAGNOSTOPULOS, G. C. SVM-based target recognition from synthetic aperture radar images using target region outline descriptors. Nonlinear Analysis, 2009, vol. 71, no. 12, p. e2934-e2939. DOI: 10.1016/j.na.2009.07.030

[7] DING, B., WEN, G., MA, C., et al. Target recognition in synthetic aperture radar images using binary morphological operations. Journal of Applied Remote Sensing, 2016, vol. 10, no. 4, p. 1-14. DOI: $10.1117 / 1 . J R S .10 .046006$
[8] HUANG, S., HUANG, W., ZHANG, T. A new SAR image segmentation algorithm for the detection of target and shadow regions. Scientific Reports, 2016, vol. 6, no. 1, p. 1-15. DOI: 10.1038/srep38596

[9] HAN, Y., LI, Y., YU, W. SAR target segmentation based on shape prior. In IEEE International Geoscience and Remote Sensing Symposium. Quebec City (Canada), 2014, p. 3738-3741. DOI: 10.1109/IGARSS.2014.6947296

[10] ZHANG, R., ZHANG, M. SAR target recognition based on active contour without edges. Journal of Systems Engineering and Electronics, 2017, vol. 28, no. 2, p. 276-281. DOI: 10.21629/JSEE.2017.02.09

[11] HU, Y., FAN, J., WANG, J. Target recognition of floating raft aquaculture in SAR image based on statistical region merging. In International Conference on Information Science and Technology. Da Nang (Vietnam), 2017, p. 429-432. DOI: 10.1109/ICIST.2017.7926798

[12] LANG, F., YANG, J., LI, D., et al. Polarimetric SAR image segmentation using statistical region merging. IEEE Geoscience and Remote Sensing Letters, 2014, vol. 11, no. 2, p. 509-513. DOI: 10.1109/LGRS.2013.2271040

[13] DA CUNHA, A. L., ZHOU, J., DO, M. N. The nonsubsampled contourlet transform: Theory, design, and applications. IEEE Transactions on Image Processing, 2006, vol. 15, no. 10, p. 3089-3101. DOI: 10.1109/TIP.2006.877507

[14] WU, K.-L., YU, J., YANG. M.-S. A novel fuzzy clustering algorithm based on a fuzzy scatter matrix with optimality tests. Pattern Recognition Letters, 2005, vol. 26, no. 5, p. 639-652. DOI: 10.1016/j.patrec.2004.09.016

[15] PEI, J., HUANG, Y., HUO, W., et al. Synthetic aperture radar processing approach for simultaneous target detection and image formation. Sensors, 2018, vol. 10, no. 18, p. 3377. DOI: 10.3390/s18103377

[16] WANG, Z., DU, L., SU, H. Target detection via Bayesianmorphological saliency in high-resolution SAR images. IEEE Transactions on Geoscience and Remote Sensing, 2017, vol. 55, no. 100, p. 5455-5466. DOI: 10.1109/TGRS.2017.2707672

[17] AMBROSANIO, M., BASELICE, F., FERRAIOLI, G., et al. Kolgomorov Smirnov test based approach for SAR automatic target recognition. In IEEE International Geoscience and Remote Sensing Symposium. Fort Worth (USA), 2017, p. 1660-1663. DOI: 10.1109/IGARSS.2017.8127292

[18] ACHANTA, R., SHAJI, A., SMITH, K., et al. SLIC superpixels compared to state-of-the-art superpixel methods. IEEE Transactions on Pattern Analysis and Machine Intelligence, 2012, vol. 34, no. 11, p. 2274-2282. DOI: 10.1109/TPAMI.2012.120

[19] LEVIN, A., LISCHINSKI, D., WEISS, Y. A closed-form solution to natural image matting. IEEE Transactions on Pattern Analysis and Machine Intelligence, 2008, vol. 30, no. 2, p. 228-242. DOI: 10.1109/TPAMI.2007.1177 
[20] KARACAN, L., ERDEM, A., ERDEM, E. Alpha matting with KL-Divergence based sparse sampling. IEEE Transactions on Image Processing, 2017, vol. 26, no. 9, p. 4523-4536. DOI: 10.1109/TIP.2017.2718664

[21] CHEN, Q., LI, D., TANG, C. K. KNN matting. IEEE Transactions on Pattern Analysis and Machine Intelligence, 2013, vol. 35, no. 9, p. 2175-2188. DOI: 10.1109/TPAMI.2013.18

[22] ROSS, T., WORRELL, S., VELTEN, V., et al. Standard SAR ATR evaluation experiments using the MSTAR public release dataset. In SPIE, Algorithms for Synthetic Aperture Radar Imagery V. Orlando (Florida), 1998, p. 566-573. DOI: 10.1117/12.321859

\section{About the Authors...}

Benish AMIN obtained her MS degree in 2015 from National University of Sciences and Technology (NUST), Pakistan. She is currently pursuing her Ph.D. degree and actively involved in image processing related research activities.

M. Mohsin RIAZ obtained his Ph.D. degree in Electrical Engineering from NUST, Pakistan, in 2013. He is serving as Assistant Professor at COMSATS Institute of Information Technology, Pakistan. His research interests include image processing and machine learning.

Abdul GHAFOOR received his Ph.D. degree from the University of Western Australia (UWA) in 2007. His research topics include model/controller order reduction, image processing/matching, through wall imaging, and cognitive radio. Since 2008, he is associated with NUST, where he is serving as an Associate Professor. 